\title{
Preoperative albumin-to-globulin ratio and prognostic nutrition index predict prognosis for glioblastoma
}

This article was published in the following Dove Press journal:

OncoTargets and Therapy

8 February 2017

Number of times this article has been viewed

\author{
Wen-Zhe $\mathrm{Xu}^{1,2}$ \\ Feng $\mathrm{Li}^{1,2}$ \\ Zhen-Kuan $\mathrm{Xu}^{1,2}$ \\ Xuan Chen ${ }^{3}$ \\ Bin Sun ${ }^{1,2}$ \\ Jing-Wei Cao ${ }^{1,2}$ \\ Yu-Guang Liu',2 \\ 'Department of Neurosurgery, \\ Qilu Hospital, ${ }^{2}$ Brain Science \\ Research Institute, ${ }^{3}$ Department of \\ Radiation Oncology, Qilu Hospital, \\ Shandong University, Jinan, People's \\ Republic of China
}

Correspondence: Yu-Guang Liu; Feng Li Department of Neurosurgery, Qilu Hospital, Shandong University, 107 Wenhuaxi Road, Jinan, Shandong 2500 I2, People's Republic of China

Tel +86 I85 6008 57I8;

+8618560085729

Email docliuyuguang@126.com; doclifeng@।26.com
Objective: Impaired immunonutritional status has disadvantageous effects on outcomes for cancer patients. Preoperative albumin-to-globulin ratio (AGR) and the prognostic nutrition index (PNI) have been used as prognostic factors in various cancers. We aimed to evaluate the clinical significance of the AGR and PNI in glioblastoma.

Materials and methods: This retrospective analysis involved 166 patients. Demographic, clinical, and laboratory data were collected. AGR and the PNI were calculated as AGR = albumin/(total serum protein - albumin) and PNI $=$ albumin $(\mathrm{g} / \mathrm{L})+5 \times$ total lymphocyte count $\left(10^{\%} / \mathrm{L}\right)$. Overall survival (OS) was estimated by Kaplan-Meier analysis. Receiver-operating characteristic analysis was used to assess the predictive ability of AGR and the PNI. Cox proportional-hazard models estimating hazard ratios (HRs) and 95\% confidence intervals (CIs) were used for univariable and multivariable survival analyses.

Results: The cutoff values of AGR and PNI were 1.75 and 48. OS was enhanced, with high AGR $(>1.75)$ and the PNI $(>48)(P<0.001$ for both). Areas under the receiver-operating characteristic curve for AGR and the PNI were 0.68 and 0.631 for 1-year survival and 0.651 and 0.656 for 2 -year survival $(P<0.05$ for all), respectively. On multivariable analyses, both AGR and the PNI were independent predictors of OS (AGR, HR 0.785, 95\% CI 0.357-0.979 $[P=0.04]$; PNI, HR $0.757,95 \%$ CI $0.378-0.985$ [ $P=0.039])$. On subgroup analysis, AGR and the PNI were significant prognostic factors for OS in patients with adjuvant therapy (AGR $P<0.001$; PNI $P=0.001$ ).

Conclusion: Preoperative AGR and the PNI may be easy-to-perform and inexpensive indices for predicting OS with glioblastoma. AGR and the PNI could also help in developing good adjuvant-therapy schedules.

Keywords: glioblastoma, albumin-to-globulin ratio, prognostic nutrition index, prognosis

\section{Introduction}

The 5-year relative survival for glioblastoma (GB), the most commonly occurring glioma histological type ( $\sim 5 \%$ of all gliomas), is $\sim 5 \% .{ }^{1}$ The widely accepted therapy regimen for newly diagnosed GB includes maximal safe resection and concurrent chemotherapy with temozolomide and radiotherapy. The median survival of GB is only about 15 months, even with recent diagnostic and therapeutic advances. ${ }^{2}$ Because of molecular and cellular heterogeneity, together with individual differences, the clinical outcomes of individual patients with similar diagnosis and treatment remain diverse. ${ }^{3}$ Moreover, a high incidence of local recurrence $(\sim 90 \%)$ is an important cause of low survival. ${ }^{4}$ Consequently, identifying prognostic biomarkers, such as in peripheral blood, could help in designing treatment strategies and stratifying patients with poor overall survival (OS) after surgery. 
Understanding the crucial biomarkers that play a part in GB malignant behavior might help clinicians educate patients about prognosis and monitor therapeutic efficacy. Laboratory markers found as prognostic factors for GB include $M G M T, I D H 1, I D H 2, E G F R$, telomerase reverse transcriptase, p53, PDGF and PTEN. ${ }^{5-9}$ Some clinical markers, such as histopathologic transformation, as well as geometric heterogeneity and/or spherical rim widths seen on magnetic resonance imaging, show high individual and combined prognostic value. ${ }^{10,11}$ However, the high cost of testing and the analytic subjectivity and instability of results limit the application of these markers in clinical practice. Novel and practical biomarkers for predicting outcomes with GB are needed.

Preoperative albumin-to-globulin ratio (AGR) could reflect both malnutrition and systemic inflammation in cancer patients. ${ }^{12}$ As a major component in serum protein, serum albumin is used to measure long-standing malnutrition and is also associated with systemic inflammation. ${ }^{13}$ Survival seems longer for patients with high than low preoperative serum albumin levels in adenocarcinoma of the gastric cardia and ovarian cancer. ${ }^{14,15}$ Globulin functions as a carrier of hormones, and plays an important role in immunity and inflammation. ${ }^{16}$ Recently, AGR was used to predict outcome with bladder urothelial carcinoma, small-cell lung cancer, and natural killer/T-cell lymphoma. ${ }^{12,17,18}$

The prognostic nutrition index (PNI), based on albumin level and lymphocyte count, could reflect immunonutritional status and systemic inflammatory reaction with more accuracy than with each variable alone. ${ }^{19,20}$ Lymphocytes are key components of the immune system and are essential effector cells in antitumor immunity. ${ }^{21,22}$ Lymphocyte count (particularly CD4 cells) has been found to be decreased in GB and associated with poor survival time..$^{23,24}$ The value of the PNI in predicting clinical outcomes has been verified in colorectal cancer, gastric cancer, and renal cell carcinoma, for example. ${ }^{25-27}$ However, no study has examined the prognostic role of AGR and the PNI in GB. In this study, we examined clinical data to analyze the prognostic value of the two immunonutritional factors AGR and PNI for OS in GB patients.

\section{Materials and methods}

\section{Patients}

We examined clinical data for 243 patients with a diagnosis of GB confirmed by postoperative pathological examination at Qilu Hospital of Shandong University from January 2010 to January 2015. Patients were followed every month for the first few years after surgery and thereafter every 2 months until death. The median follow-up time was 14 months (range 1-52 months). We excluded patients with metabolic syndrome, diabetes, heart disease, hypertension, autoimmune disease, and infection within 3 months.

We collected data on age, sex, smoking and drinking status, body mass index, Karnofsky performance status (KPS), tumor size, tumor localization (region of brain), extent of resection, preoperative lymphocyte count, preoperative albumin level, adjuvant radiotherapy and chemotherapy, and death. Tumor size was calculated from magnetic resonanceimaging data: longest $\times$ widest diameter $\times$ thickness (section thickness $\times$ number of layers) $\times 0.5 .^{28}$

Data for hematological variables were obtained for all enrolled patients within 1 week before surgery. The time from surgery to death was defined as OS. Data for patients alive at last follow-up were considered censored. The study protocol was approved by the ethics committee of Qilu Hospital. Patients all granted written informed consent for surgery and the use of clinical records.

\section{Evaluation of AGR and PNI}

We used the following formulas for calculating AGR and PNI: ${ }^{29}$

$\mathrm{AGR}=$ Albumin/(total serum protein - albumin $)$

$\mathrm{PNI}=$ Albumin $(\mathrm{g} / \mathrm{L})+5 \times$ Total lymphocyte count $\left(10^{9} / \mathrm{L}\right)$

\section{Statistical analysis}

To determine differences between groups, continuous variables were analyzed by unpaired $t$-tests and categorical variables by $\chi^{2}$ test. Data are shown as means (standard deviation) or median (interquartile range). We used the Kaplan-Meier method for estimating OS. Receiver-operating characteristic (ROC) curve analysis was applied to determine the best cutoff values for AGR and PNI, and area under the ROC curve estimation was used to assess the predictive ability of AGR and PNI. Univariable Cox analysis was used to analyze the prognostic significance of the variables. Multivariable analysis by Cox proportional-hazard model was used to determine the independence of the prognostic factors, estimating hazard ratios (HRs) and 95\% confidence intervals (CIs). All statistical analyses involved the use of Statistical Package for the Social Sciences (SPSS) version 23.0 (IBM, Armonk, NY, USA). $P<0.05$ was considered statistically 
significant and the inclusion criterion to use a variable from univariable analysis in the multivariable analysis.

\section{Results}

\section{Clinical characteristics of patients}

After excluding data for 65 patients with the aforementioned diseases and 12 patients lost to follow-up, we examined data for 166 patients. Baseline characteristics are in Table 1 . The median age at diagnosis was $50(6-83)$ years, and $84(50.6 \%)$ were male. The median body mass index was 22 (15.57-34.38). KPS was $80-100$ in 91 patients (54.8\%) and $\leq 70$ in 75 patients $(45.2 \%)$. The median tumor size was $40.1(6.35-245.52) \mathrm{cm}^{3}$. Most tumors were located in the frontal (38.6\%), temporal (33.7\%), and parieto-occipital

Table I Clinical characteristics of I 66 patients with glioblastoma

\begin{tabular}{|c|c|}
\hline Age (years), mean \pm SD & $50.4 I \pm 14.5$ \\
\hline Median (IQR) & $50(6-83)$ \\
\hline Sex (male/female) & $84 / 82$ \\
\hline Preoperative BMI, mean \pm SD & $22.4 \pm 3.52$ \\
\hline Median (IQR) & $22(15.57-34.38)$ \\
\hline \multicolumn{2}{|l|}{ KPS } \\
\hline $80-100$ & 91 (54.8\%) \\
\hline$\leq 70$ & 75 (45.2\%) \\
\hline \multicolumn{2}{|l|}{ Smoking } \\
\hline Never or light & $115(69.3 \%)$ \\
\hline Heavy & $5 \mathrm{I}(30.7 \%)$ \\
\hline \multicolumn{2}{|l|}{ Drinking } \\
\hline Never or light & $122(73.5 \%)$ \\
\hline Heavy & $44(26.5 \%)$ \\
\hline Tumor size $\left(\mathrm{cm}^{3}\right)$, mean \pm SD & $52.61 \pm 28.12$ \\
\hline Median (IQR) & $40.1(6.35-245.52)$ \\
\hline \multicolumn{2}{|l|}{ Tumor location, region } \\
\hline Frontal & $64(38.6 \%)$ \\
\hline Temporal & $56(33.7 \%)$ \\
\hline Parieto-occipital & $34(20.5 \%)$ \\
\hline Other & $12(7.2 \%)$ \\
\hline \multicolumn{2}{|l|}{ Extent of resection } \\
\hline Total & $109(65.7 \%)$ \\
\hline Not total & $57(34.3 \%)$ \\
\hline \multicolumn{2}{|l|}{ Preoperative } \\
\hline Albumin level (g/L), mean $\pm S D$ & $42.21 \pm 4.07$ \\
\hline Median (IQR) & $41.6 I(33.91-52.11)$ \\
\hline Lymphocyte count $\left(10^{9} / \mathrm{L}\right)$, mean \pm SD & $1.97 \pm 0.64$ \\
\hline Median (IQR) & $1.89(0.73-3.67)$ \\
\hline AGR, mean \pm SD & $1.65 \pm 0.41$ \\
\hline Median (IQR) & $1.66(1.19-2.58)$ \\
\hline PNI, mean \pm SD & $51.92 \pm 5.23$ \\
\hline Median (IQR) & $51.1(41.15-68.95)$ \\
\hline Adjuvant radiotherapy & $105(63.3 \%)$ \\
\hline Adjuvant chemotherapy & $42(25.3 \%)$ \\
\hline
\end{tabular}

Note: Data are $\mathrm{n}(\%)$, unless otherwise indicated.

Abbreviations: SD, standard deviation; IQR, interquartile range; BMI, body mass index; KPS, Karnofsky performance status; AGR, albumin-to-globulin ratio; PNI, prognostic nutrition index.
(20.5\%) regions. Other locations, including ventricles, cerebellum, insular lobe, thalamus, and callosum, represented $7.2 \%$ of tumors. Total resection was performed to treat 109 patients $(65.7 \%)$. The median preoperative albumin level, lymphocyte count, AGR, and PNI were 41.61 (33.91-52.11) g/L, $1.89 \times 10^{9} / \mathrm{L}(0.73-3.67), 1.66(1.19-2.58)$, and 51.1 (41.15-68.95), respectively. In all, 105 patients (63.3\%) received adjuvant radiotherapy and $42(25.3 \%)$ adjuvant chemotherapy.

\section{Association of clinical characteristics with preoperative AGR and PNI}

On ROC analysis, the cutoff values for AGR and PNI were 1.75 (sensitivity 0.554 and specificity 0.806 ) and 48 (sensitivity 0.782 and specificity 0.575 ). The high-AGR and -PNI groups had higher preoperative albumin levels than the low-AGR and -PNI groups ( $P<0.001$ for both) (Table 2). Similarly, preoperative lymphocyte counts in the high-AGR and -PNI groups were higher than those in the low-AGR and -PNI groups $(P=0.013$ and $P<0.001)$.

\section{Prognostic value of AGR and PNI}

The median OS was 13 (range 1-52) months. KaplanMeier curves for OS based on preoperative AGR and PNI demonstrated that patients with AGR $\leq 1.75$ or PNI $\leq 48$ had significantly poorer OS than those with AGR $>1.75$ or PNI $>48(P<0.001$ for both) (Figure 1). For 1-year survival, the area under the ROC curve for AGR and PNI was 0.68 (sensitivity 0.67 , specificity $0.774,95 \%$ CI $0.577-0.783$; $P=0.038$ ), and 0.631 (sensitivity 0.834 , specificity $0.429,95 \%$ CI $0.523-0.739 ; P=0.045$ ), and for 2-year survival was 0.651 (sensitivity 0.524 , specificity $0.786,95 \%$ CI $0.55-0.752$; $P=0.024$ ) and 0.656 (sensitivity 0.766 , specificity $0.55,95 \%$ CI 0.548-0.765; $P=0.041$ ) (Figure 2).

\section{Univariable and multivariable analyses of prognostic factors for OS}

We examined the functions of preoperative AGR and PNI in survival in our cohort (Table 3). On univariable Cox analysis, OS was associated with age $(P=0.045)$, KPS $(P=0.009)$, tumor size $(P=0.022)$, extent of resection ( $P=0.017)$, preoperative albumin level $(P=0.035)$, lymphocyte count $(P=0.028)$, AGR $(P=0.003)$, PNI $(P<0.001)$, and adjuvant radiotherapy $(P=0.004)$ and chemotherapy $(P=0.002)$. On multivariable analysis, predictors of OS were KPS (HR 0.528, 95\% CI 0.341-0.851; $P=0.032$ ), tumor size (HR 1.343, 95\% CI 1.014-1.726; $P=0.037$ ), extent of resection (HR $0.416,95 \%$ CI $0.334-0.885 ; P=0.026$ ), preoperative 
Table 2 Clinical characteristics of patients by AGR and PNI cutoffs

\begin{tabular}{|c|c|c|c|c|c|c|}
\hline \multirow[t]{2}{*}{ Characteristics } & \multicolumn{3}{|l|}{ AGR } & \multicolumn{3}{|l|}{ PNI } \\
\hline & $\leq 1.75$ & $>1.75$ & $P$-value & $\leq 48$ & $>48$ & $P$-value \\
\hline Age, years & $50.5 \pm 14.94$ & $50.39 \pm 11.68$ & 0.958 & $52.74 \pm 14.06$ & $49.29 \pm 14.59$ & 0.134 \\
\hline Sex & & & 0.496 & & & 0.391 \\
\hline Male & 52 & 33 & & 28 & 56 & \\
\hline Female & 51 & 30 & & 30 & 52 & \\
\hline Preoperative BMI & $22.67 \pm 3.89$ & $22.1 \pm 2.97$ & 0.293 & $21.58 \pm 3.91$ & $22.44 \pm 4.04$ & 0.178 \\
\hline KPS & & & 0.335 & & & 0.87 \\
\hline $80-100$ & 53 & 38 & & 31 & 60 & \\
\hline$\leq 70$ & 50 & 25 & & 27 & 48 & \\
\hline Smoking & & & 0.145 & & & 0.542 \\
\hline Never or light & 69 & 46 & & 40 & 75 & \\
\hline Heavy & 34 & 17 & & 18 & 33 & \\
\hline Drinking & & & 0.155 & & & 0.377 \\
\hline Never or light & 79 & 43 & & 44 & 78 & \\
\hline Heavy & 24 & 20 & & 14 & 30 & \\
\hline Tumor size $\left(\mathrm{cm}^{3}\right)$ & $54.25 \pm 44.37$ & $50.13 \pm 37.55$ & 0.528 & $53.83 \pm 36.97$ & $51.92 \pm 46.37$ & 0.77 \\
\hline Tumor location & & & 0.522 & & & 0.191 \\
\hline Frontal & 44 & 19 & & 27 & 37 & \\
\hline Temporal & 33 & 24 & & 16 & 40 & \\
\hline Parieto-occipital & 20 & 14 & & 9 & 25 & \\
\hline Other & 7 & 5 & & 6 & 6 & \\
\hline Extent of resection & & & 0.108 & & & 0.491 \\
\hline Total & 63 & 46 & & 35 & 74 & \\
\hline Not total & 40 & 17 & & 22 & 35 & \\
\hline Preoperative albumin level (g/L) & $40.34 \pm 3.34$ & $43.20 \pm 4.35$ & $<0.001$ & $41.09 \pm 3.28$ & $43.69 \pm 4.41$ & $<0.001$ \\
\hline Preoperative lymphocyte count (109/L) & $1.91 \pm 0.55$ & $2.10 \pm 0.61$ & 0.013 & $1.87 \pm 0.46$ & $2.22 \pm 0.48$ & $<0.001$ \\
\hline Adjuvant radiotherapy & & & 0.192 & & & 0.093 \\
\hline No & 24 & 37 & & 34 & 27 & \\
\hline Yes & 50 & 55 & & 46 & 59 & \\
\hline Adjuvant chemotherapy & & & 0.173 & & & 0.269 \\
\hline No & 80 & 44 & & 53 & 71 & \\
\hline Yes & 23 & 19 & & 15 & 27 & \\
\hline
\end{tabular}

Note: Data are mean \pm SD.

Abbreviations: AGR, albumin-to-globulin ratio; PNI, prognostic nutrition index; BMI, body mass index; KPS, Karnofsky performance status; SD, standard deviation.

A

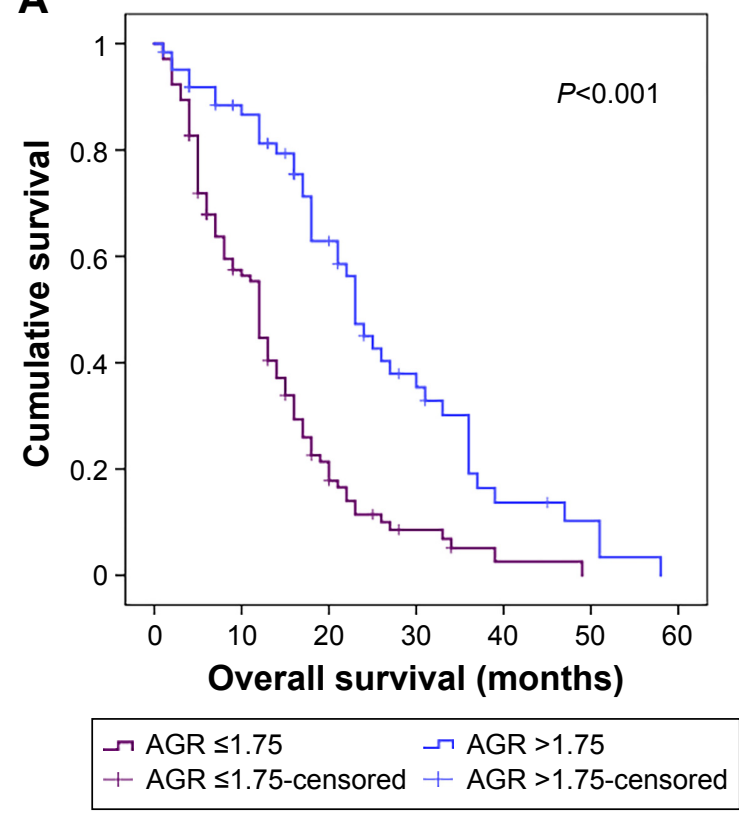

B
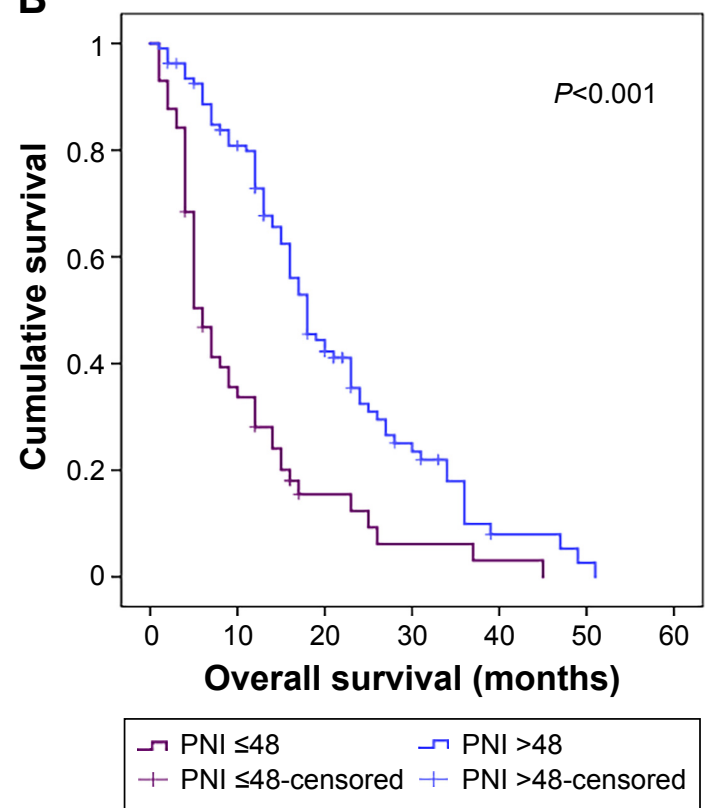

Figure I Prognosis of preoperative (A) AGR and (B) PNI in survival with glioblastoma. Abbreviations: AGR, albumin-to-globulin ratio; PNI, prognostic nutrition index. 

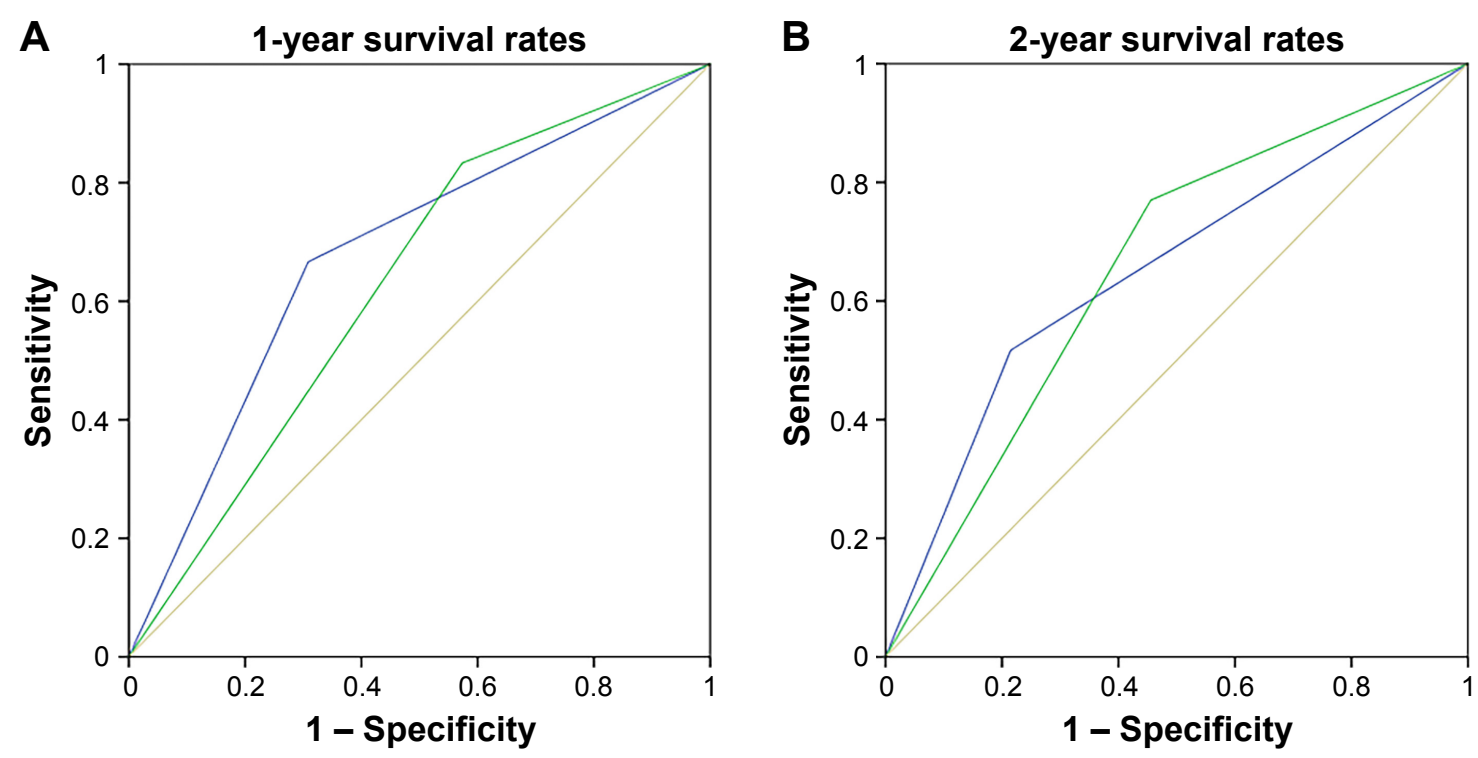

AGR $\longrightarrow$ PNI $\longrightarrow$ Reference line

Figure 2 Predictive value of AGR and PNI for (A) I-year and (B) 2-year survival. Receiver-operating characteristic curves for AGR and PNI. Abbreviations: AGR, albumin-to-globulin ratio; PNI, prognostic nutrition index.

AGR (HR 0.785, 95\% CI 0.357-0.979; $P=0.04$ ), PNI (HR $0.757,95 \%$ CI $0.378-0.985 ; P=0.039)$, and adjuvant radiotherapy (HR 0.599, 95\% CI 0.32-0.983; $P=0.031$ ).

\section{Prognostic impact of AGR and PNI with adjuvant therapy}

Adjuvant therapy was defined as chemotherapy and/or radiotherapy. In our cohort, $123(74.1 \%)$ patients received adjuvant therapy. On subgroup analyses of patients with adjuvant therapy, survival differed with AGR $\leq 1.75$ and $>1.75(P<0.001)$ (Figure $3 \mathrm{~A})$ and $\mathrm{PNI} \leq 48$ and $>48$ $(P=0.001)$ (Figure 3B).

\section{Discussion}

Impaired immunonutritional status has disadvantageous effects on outcomes for cancer patients. AGR and the PNI

Table 3 Prognostic factors for overall survival for 166 patients with glioblastoma

\begin{tabular}{|c|c|c|c|c|c|c|}
\hline \multirow[t]{2}{*}{ Characteristics } & \multicolumn{2}{|c|}{ Univariable analysis } & \multirow[t]{2}{*}{$P$-value } & \multicolumn{2}{|c|}{$\begin{array}{l}\text { Multivariable } \\
\text { analysis }\end{array}$} & \multirow[t]{2}{*}{ P-value } \\
\hline & HR & $95 \% \mathrm{Cl}$ & & HR & $95 \% \mathrm{Cl}$ & \\
\hline Age $(>\mathbf{5 0}$ vs $\leq \mathbf{5 0}$ years $)$ & 1.423 & $1.00 \mathrm{I}-2.145$ & 0.045 & 1.017 & $0.996-2.286$ & 0.371 \\
\hline Sex (female vs male) & 1.014 & $0.718-1.434$ & 0.935 & & & \\
\hline Preoperative BMI ( $>22$ vs $\left.\leq 22 \mathrm{~kg} / \mathrm{m}^{2}\right)$ & I & $0.859-1.142$ & 0.993 & & & \\
\hline KPS (80- 100 vs $\leq 70)$ & 0.403 & $0.257-0.694$ & 0.009 & 0.528 & $0.34 I-0.85 I$ & 0.032 \\
\hline Smoking (heavy vs never or light) & 1.096 & $0.76 I-1.577$ & 0.623 & & & \\
\hline Drinking (heavy vs never or light) & 0.944 & $0.645-1.383$ & 0.769 & & & \\
\hline Tumor size $\left(>40\right.$ vs $\left.\leq 40 \mathrm{~cm}^{3}\right)$ & 1.769 & $1.41-2.198$ & 0.022 & 1.343 & $1.014-1.726$ & 0.037 \\
\hline \multicolumn{7}{|l|}{ Tumor location } \\
\hline Frontal & & I & Ref & & & \\
\hline Temporal & 1.153 & $0.804-1.656$ & 0.49 & & & \\
\hline Parieto-occipital & 0.761 & $0.44-1.237$ & 0.271 & & & \\
\hline Other & 1.294 & $0.713-2.476$ & 0.363 & & & \\
\hline Extent of resection (total vs not total) & 0.315 & $0.244-0.762$ & 0.017 & 0.416 & $0.334-0.885$ & 0.026 \\
\hline Preoperative albumin level ( $>42$ vs $\leq 42$ g/L) & 0.617 & $0.374-0.812$ & 0.035 & 0.918 & $0.57 I-1.389$ & 0.359 \\
\hline Preoperative lymphocyte count ( $>1.9$ vs $\left.\leq 1.9 \times 10^{9} / \mathrm{L}\right)$ & 0.578 & $0.379-0.961$ & 0.028 & 0.84 & $0.54 I-1.225$ & 0.196 \\
\hline $\operatorname{AGR}(>I .75$ vs $\leq 1.75)$ & 0.456 & $0.244-0.608$ & 0.003 & 0.785 & $0.357-0.979$ & 0.04 \\
\hline PNI $(>48$ vs $\leq 48)$ & 0.372 & $0.245-0.565$ & $<0.001$ & 0.757 & $0.378-0.985$ & 0.039 \\
\hline Adjuvant radiotherapy (yes vs no) & 0.554 & $0.402-0.721$ & 0.004 & 0.599 & $0.320-0.983$ & 0.031 \\
\hline Adjuvant chemotherapy (yes vs no) & 0.542 & $0.357-0.822$ & 0.002 & 0.866 & $0.494-1.298$ & 0.226 \\
\hline
\end{tabular}

Abbreviations: HR, hazard ratio; Cl, confidence interval; BMI, body mass index; KPS, Karnofsky performance status; AGR, albumin-to-globulin ratio; PNI, prognostic nutrition index. 
A

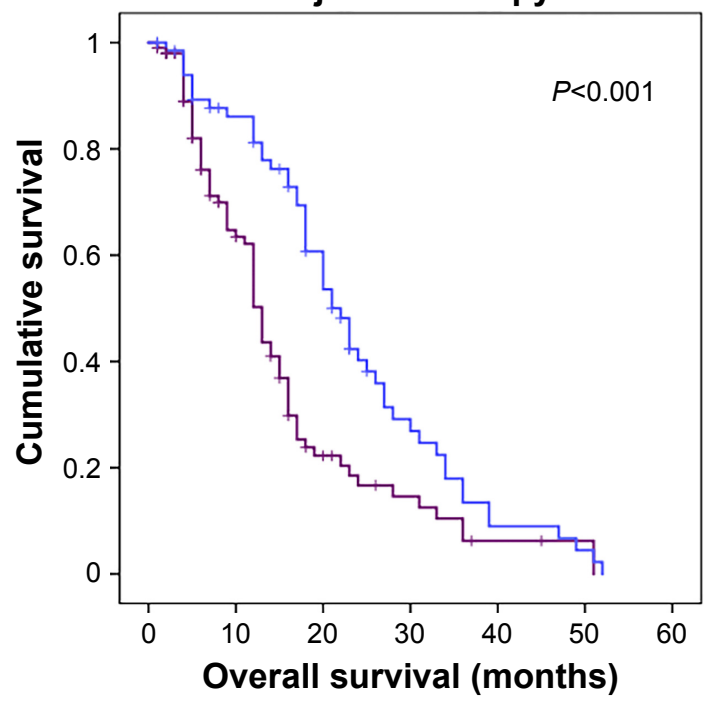

\begin{tabular}{ll|}
$\rightarrow$ AGR $\leq 1.75$ & $\neg$ AGR $>1.75$ \\
+ AGR $\leq 1.75$-censored & + AGR $>1.75$-censored \\
\hline
\end{tabular}
B

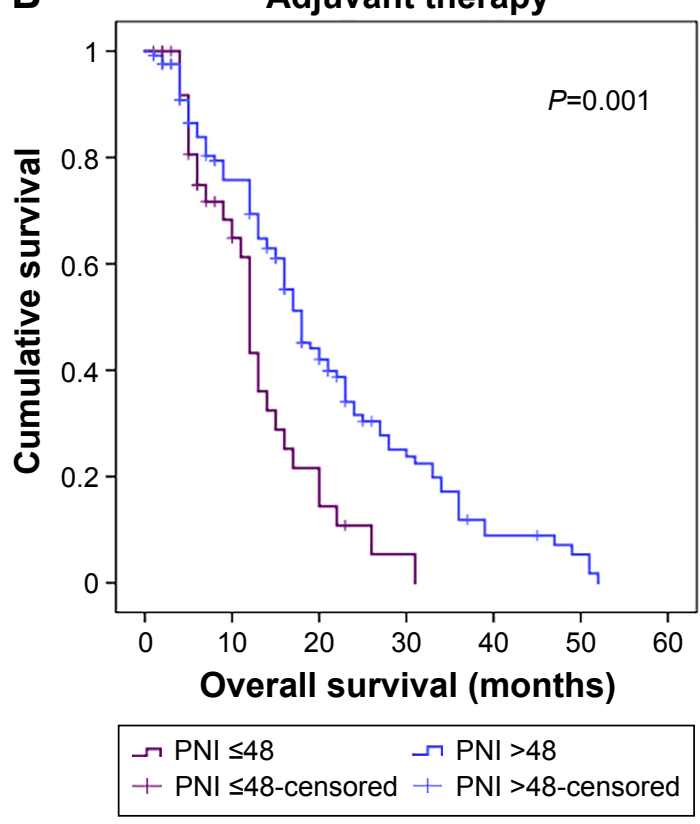

Figure 3 Subgroup analyses of the prognostic impact of (A) AGR and (B) PNI for patients with adjuvant therapy by Kaplan-Meier survival curves. Abbreviations: AGR, albumin-to-globulin ratio; PNI, prognostic nutrition index.

have been used as prognostic factors in various cancers. We evaluated the clinical significance of AGR and the PNI in 166 patients with GB. OS was enhanced with high AGR $(>1.75)$ and PNI $(>48)$. Both were independent predictors of OS, and significant prognostic factors for OS in patients with adjuvant therapy. Preoperative AGR and PNI may be easy-to-perform and inexpensive indices for predicting OS in GB patients.

Cancer patients frequently show malnutrition, which is responsible for decreased capacity for treatment tolerance, increased mortality, and worse quality of life in the postoperative period..$^{30}$ Moreover, malnutrition has been found to be associated with an immunosuppressed condition, ${ }^{31}$ which suggests inadequate antitumor immunity. ${ }^{21}$ Chronic inflammation has been identified as a hallmark of cancer, and most neoplastic tissues contain an inflammatory component, even those not causally associated with an obvious inflammatory reaction. ${ }^{32}$ Cell inflammatory mediators promote tumorigenesis by increasing tumor proliferation and metastasis, and can also exert an immunosuppressive effect, with resulting impaired lymphocyte function and reduced lymphocyte count, which enables the escape of cancer cells from immunosurveillance. ${ }^{33-35}$ Meanwhile, inflammation was found to be closely related to the genesis and development of GB. ${ }^{23,24,28,36}$ In view of the relationship between nutrition and immunocompetence, as well as the influence of inflammation on GB, patients' nutritional status and immunocompetence should be evaluated in the management of GB.
In this study, we evaluated AGR and the PNI, two integrated immunonutritional indices, for their prognostic values for GB patients, and found cutoff values of 1.75 and 48 , respectively. Preoperative AGR and the PNI were significantly associated with OS in GB, which agrees with findings for other malignancies. ${ }^{12,17,18,25-27}$ Although AGR and PNI were associated with preoperative albumin level and lymphocyte count, the predictive value of both remained significant after adjustment for other clinical characteristics on multivariable analysis. According to the ROC analysis, AGR had better specificity than the PNI, and the PNI had better sensitivity than AGR. The two factors may accurately predict OS with GB. Based on indicators of preoperative inflammation, immunity, and nutritional status, AGR and the PNI could provide important prognostic information in GB.

The good prognosis with high AGR and PNI values might be explained by the functions of albumin, globulin, and lymphocytes. Albumin has antioxidative effects against some of the most common carcinogens, including nitrosamines and aflatoxins. Also, it can stabilize cell growth and DNA replication and buffer sex-hormone homeostasis to prevent sex hormone-induced cancers. ${ }^{37}$ Albumin can inhibit the proliferation of human breast cancer cells by regulating the activities of autocrine growth-regulatory factors in vitro. ${ }^{38}$ However, the inflammatory response was reported to play an independent role in decreasing albumin level apart from malnutrition. ${ }^{13}$ In cancer-related 
inflammation, the proinflammatory cytokines released by tumor and immune cells modulate albumin production and help in cancer progression, angiogenesis, and malignant transformation. ${ }^{32,39}$ Serum globulin, including immunoglobulins, CRP, complements, and other acutephase proteins are upregulated with inflammation and play a crucial role in immunity and inflammation. ${ }^{40,41}$ As a component of the innate immune system, high CRP levels can induce the production of inflammatory cytokines and tissue factor in monocytes. ${ }^{42}$ Complement activation can initiate or sustain the inflammatory process by leading to chemotaxis, plasma protein exudation at inflammatory sites, and opsonization of infectious agents and damaged cells. ${ }^{41}$ High levels of globulin reflect an inflammatory state, believed to play a major role in cancer progression and metastasis. ${ }^{33}$ Lymphocytes play a key role in host defense against cancer by inhibiting the proliferation and invasion of tumor cells via inducing cytotoxic cell death and production of cytokines. ${ }^{32}$ Cytotoxic T lymphocytes inhibit tumor growth by inducing apoptosis of cancer cells, whereas $\mathrm{CD}^{+}$ T-lymphocyte infiltration is associated with overall good outcome. ${ }^{43}$ Lymphocytopenia, reflecting insufficient antitumor immunocompetence, can be induced by a systemic inflammatory reaction. ${ }^{20}$

We performed a subgroup analysis of OS for patients with adjuvant therapy (radiotherapy and/or chemotherapy). For GB patients receiving adjuvant therapy after surgery, low AGR or PNI value was significantly associated with short OS, so these patients acquired less benefit from adjuvant therapy. Patients with poor immune and nutrition status showed inferior tolerance to adjuvant therapy, and the adverse effects of adjuvant therapy were more prominent in patients with immunosuppression and malnutrition, thus affecting the long-term outcomes. ${ }^{30,44}$ Albumin could protect tissues against most forms of radiation, ${ }^{37}$ thereby protecting against the adverse effects of radiotherapy. Moreover, systemic inflammation might impair the activity of CYP3A4, a crucial drug-metabolizing enzyme. ${ }^{45}$ Decreased activity of CYP3A4 has been found to be related to reduced hepatic drug clearance and increased drug toxicity. ${ }^{46}$ Therefore, it is important to maximize the curative effects of adjuvant therapy and diminish its adverse effects.

AGR and the PNI may be appropriate factors for survival in stratifying patients who acquire more benefit from adjuvant therapy, and immunonutritional intervention and anti-inflammation therapy could be considered efficient therapeutic strategies for GB patients who have received radiotherapy and/or chemotherapy.
The present study has several limitations. First, the retrospective design and limited number of patients might indicate bias. Second, unknown physiological and pathophysiological factors might have had an unavoidable impact on AGR and the PNI. Furthermore, although serum CRP and cytokine levels were important indicators of cancer-related inflammation, these factors were not routinely examined in our hospital, which prevents us from acquiring a better understanding of the mechanism of tumor progression caused by inflammation. In this study, AGR and the PNI were assessed only once before surgery. The predictive significance of the kinetics of AGR and the PNI remains to be investigated.

\section{Conclusion}

This study demonstrated that low AGR and PNI values could be promising immunonutritional markers to identify GB patients with poor prognostic potential. The application of AGR and the PNI in evaluating preoperative immunonutritional status might facilitate the formulation of more effective preoperative regulation strategies and better adjuvant-therapy schedules.

\section{Acknowledgments}

This work was supported by the National Natural Science Foundation of China (81301127 and 81401867), the China Postdoctoral Science Foundation (2014T70661 and 2014M560562), and the Key Research and Development Program of Shandong Province (2015GSF118095).

\section{Disclosure}

The authors report no conflicts of interest in this work.

\section{References}

1. Ostrom QT, Bauchet L, Davis FG, et al. The epidemiology of glioma in adults: a "state of the science" review. Neuro Oncol. 2014;16(7): 896-913.

2. Stupp R, Mason WP, van den Bent MJ, et al. Radiotherapy plus concomitant and adjuvant temozolomide for glioblastoma. $N$ Engl J Med. 2005; 352(10):987-996.

3. Aum DJ, Kim DH, Beaumont TL, Leuthardt EC, Dunn GP, Kim AH. Molecular and cellular heterogeneity: the hallmark of glioblastoma. Neurosurg Focus. 2014;37(6):E11.

4. Nabors LB, Portnow J, Ammirati M, et al. Central Nervous System Cancers, Version 1.2015. J Natl Compr Canc Netw. 2015;13(10):1191-1202.

5. Wick W, Platten M, Meisner C, et al. Temozolomide chemotherapy alone versus radiotherapy alone for malignant astrocytoma in the elderly: the NOA-08 randomised, phase 3 trial. Lancet Oncol. 2012;13(7): 707-715.

6. Idoate MA, Echeveste J, Diez-Valle R, Lozano MD, Aristu J. Biological and clinical significance of the intratumour heterogeneity of PTEN protein expression and the corresponding molecular abnormalities of the PTEN gene in glioblastomas. Neuropathol Appl Neurobiol. 2014; 40(6):736-746. 
7. Labussiere M, Boisselier B, Mokhtari K, et al. Combined analysis of TERT, EGFR, and IDH status defines distinct prognostic glioblastoma classes. Neurology. 2014;83(13):1200-1206.

8. Michalet S, Rohr J, Warshan D, et al. Phytochemical analysis of mature tree root exudates in situ and their role in shaping soil microbial communities in relation to tree $\mathrm{N}$-acquisition strategy. Plant Physiol Biochem. 2013;72:169-177.

9. Phillips JJ, Aranda D, Ellison DW, et al. PDGFRA amplification is common in pediatric and adult high-grade astrocytomas and identifies a poor prognostic group in IDH1 mutant glioblastoma. Brain Pathol. 2013; 23(5):565-573.

10. Ho AL, Koch MJ, Tanaka S, et al. Impact of histopathological transformation and overall survival in patients with progressive anaplastic glioma. J Clin Neurosci. 2016;31:99-105.

11. Perez-Beteta J, Martinez-Gonzalez A, Molina D, et al. Glioblastoma: does the pre-treatment geometry matter? A postcontrast T1 MRI-based study. Eur Radiol. Epub 2016 Jun 21.

12. Liu J, Dai Y, Zhou F, et al. The prognostic role of preoperative serum albumin/globulin ratio in patients with bladder urothelial carcinoma undergoing radical cystectomy. Urol Oncol. 2016;34(11): 484.e1-484.e8.

13. McMillan DC, Watson WS, O'Gorman P, Preston T, Scott HR, McArdle CS. Albumin concentrations are primarily determined by the body cell mass and the systemic inflammatory response in cancer patients with weight loss. Nutr Cancer. 2001;39(2):210-213.

14. Asher V, Lee J, Bali A. Preoperative serum albumin is an independent prognostic predictor of survival in ovarian cancer. Med Oncol. 2012; 29(3):2005-2009.

15. Lien YC, Hsieh CC, Wu YC, et al. Preoperative serum albumin level is a prognostic indicator for adenocarcinoma of the gastric cardia. J Gastrointest Surg. 2004;8(8):1041-1048.

16. Zhang B, Yu W, Zhou LQ, et al. Prognostic significance of preoperative albumin-globulin ratio in patients with upper tract urothelial carcinoma. PLoS One. 2015;10(12):e0144961.

17. Zhou T, He X, Fang W, et al. Pretreatment albumin/globulin ratio predicts the prognosis for small-cell lung cancer. Medicine (Baltimore). 2016; 95(12):e3097.

18. Bi XW, Wang L, Zhang WW, et al. The pretreatment albumin to globulin ratio predicts survival in patients with natural killer/T-cell lymphoma. PeerJ. 2016;4:e1742.

19. Okamura Y, Ashida R, Ito T, Sugiura T, Mori K, Uesaka K. Preoperative neutrophil to lymphocyte ratio and prognostic nutritional index predict overall survival after hepatectomy for hepatocellular carcinoma. World J Surg. 2015;39(6):1501-1509.

20. Hong S, Zhou T, Fang W, et al. The prognostic nutritional index (PNI) predicts overall survival of small-cell lung cancer patients. Tumour Biol.2015; 36(5):3389-3397.

21. Mellman I, Coukos G, Dranoff G. Cancer immunotherapy comes of age. Nature. 2011;480(7378):480-489.

22. Shibutani M, Maeda K, Nagahara $H$, et al. The prognostic significance of the postoperative prognostic nutritional index in patients with colorectal cancer. BMC Cancer. 2015;15:521.

23. Waziri A. Glioblastoma-derived mechanisms of systemic immunosuppression. Neurosurg Clin N Am. 2010;21(1):31-42.

24. Gustafson MP, Lin Y, LaPlant B, et al. Immune monitoring using the predictive power of immune profiles. J Immunother Cancer. 2013;1:7.

25. Yang Y, Gao P, Chen X, et al. Prognostic significance of preoperative prognostic nutritional index in colorectal cancer: results from a retrospective cohort study and a meta-analysis. Oncotarget. 2016;7(36): $58543-58552$

26. Wang SH, Zhai ST, Lin H. Role of prognostic nutritional index in patients with gastric cancer: a meta-analysis. Minerva Med. 2016;107(5): $322-327$.
27. Grimes N, Tyson M, Hannan C, Mulholland C. A systematic review of the prognostic role of hematologic scoring systems in patients with renal cell carcinoma undergoing nephrectomy with curative intent. Clin Genitourin Cancer. 2016;14(4):271-276.

28. Han S, Liu Y, Li Q, Li Z, Hou H, Wu A. Pre-treatment neutrophil-to-lymphocyte ratio is associated with neutrophil and T-cell infiltration and predicts clinical outcome in patients with glioblastoma. BMC Cancer. 2015; $15: 617$.

29. Pinato DJ, North BV, Sharma R. A novel, externally validated inflammation-based prognostic algorithm in hepatocellular carcinoma: the prognostic nutritional index (PNI). Br J Cancer. 2012;106(8): 1439-1445.

30. Kanda M, Mizuno A, Tanaka C, et al. Nutritional predictors for postoperative short-term and long-term outcomes of patients with gastric cancer. Medicine (Baltimore). 2016;95(24):e3781.

31. Mainous MR, Deitch EA. Nutrition and infection. Surg Clin North Am. 1994;74(3):659-676.

32. Mantovani A, Allavena P, Sica A, Balkwill F. Cancer-related inflammation. Nature. 2008;454(7203):436-444.

33. Colotta F, Allavena P, Sica A, Garlanda C, Mantovani A. Cancer-related inflammation, the seventh hallmark of cancer: links to genetic instability. Carcinogenesis. 2009;30(7):1073-1081.

34. Kusmartsev S, Gabrilovich DI. Immature myeloid cells and cancerassociated immune suppression. Cancer Immunol Immunother. 2002; 51(6):293-298.

35. Salazar-Onfray F, Lopez MN, Mendoza-Naranjo A. Paradoxical effects of cytokines in tumor immune surveillance and tumor immune escape. Cytokine Growth Factor Rev. 2007;18(1-2):171-182.

36. Bambury RM, Teo MY, Power DG, et al. The association of pretreatment neutrophil to lymphocyte ratio with overall survival in patients with glioblastoma multiforme. J Neurooncol. 2013;114(1):149-154.

37. Seaton K. Albumin concentration controls cancer. J Natl Med Assoc. 2001;93(12):490-493.

38. Laursen I, Briand P, Lykkesfeldt AE. Serum albumin as a modulator on growth of the human breast cancer cell line, MCF-7. Anticancer Res. 1990; 10(2A):343-351.

39. Rothschild MA, Oratz M, Schreiber SS. Serum albumin. Hepatology. 1988;8(2):385-401.

40. Li Q, Meng X, Liang L, Xu Y, Cai G, Cai S. High preoperative serum globulin in rectal cancer treated with neoadjunctive chemoradiation therapy is a risk factor for poor outcome. Am J Cancer Res. 2015; 5(9):2856-2864.

41. Gabay C, Kushner I. Acute-phase proteins and other systemic responses to inflammation. N Engl J Med. 1999;340(6):448-454.

42. Ballou SP, Lozanski G. Induction of inflammatory cytokine release from cultured human monocytes by C-reactive protein. Cytokine. 1992; 4(5):361-368

43. Fu Y, Chen SW, Chen SQ, et al. A preoperative nutritional index for predicting cancer-specific and overall survival in Chinese patients with laryngeal cancer: a retrospective study. Medicine (Baltimore). 2016; 95(11): 2962

44. Cai B, Chen H, Sun H, Wan P, Sun H, Pan J. Production of immunoregulatory polysaccharides from Crassostrea hongkongensis and their positive effects as a nutrition factor in modulating the effectiveness and toxicity of 5-FU chemotherapy in mice. Food Funct. 2016;7(1): 390-397.

45. Charles KA, Rivory LP, Brown SL, Liddle C, Clarke SJ, Robertson GR. Transcriptional repression of hepatic cytochrome P450 3A4 gene in the presence of cancer. Clin Cancer Res. 2006;12(24):7492-7497.

46. Rivory LP, Slaviero KA, Clarke SJ. Hepatic cytochrome P450 3A drug metabolism is reduced in cancer patients who have an acute-phase response. Br J Cancer. 2002;87(3):277-280. 
OncoTargets and Therapy

\section{Publish your work in this journal}

OncoTargets and Therapy is an international, peer-reviewed, open access journal focusing on the pathological basis of all cancers, potential targets for therapy and treatment protocols employed to improve the management of cancer patients. The journal also focuses on the impact of management programs and new therapeutic agents and protocols on

patient perspectives such as quality of life, adherence and satisfaction. The manuscript management system is completely online and includes a very quick and fair peer-review system, which is all easy to use. Visit http://www.dovepress.com/testimonials.php to read real quotes from published authors.

Submit your manuscript here: http://www.dovepress.com/oncotargets-and-therapy-journal 